\title{
Utilization, receptivity and reactivity to Interactive Voice Response daily monitoring in risky drinking smokers who are motivated to quit
}

\author{
Amy M. Cohn', Hoda Elmasry², Sarah J. Ehlke
}

\begin{abstract}
INTRODUCTION Interactive Voice Response (IVR) technology has become an increasingly popular and valid method for collecting Ecological Momentary Assessment (EMA) data on a variety of health-risk behaviors, including daily alcohol use and cigarette smoking, and for stimulating behavior change. However, very little research has evaluated the parameters of IVR compliance and reactivity in respondents who may have greater problem severity than samples previously examined in published IVR studies. This study examined the prevalence and correlates of use, receptivity and reactivity to IVR monitoring in 77 untreated risky drinking smokers who were motivated to quit within the next 6 months.

METHODS Respondents completed twice daily IVR assessments for 28 days and were re-assessed immediately after IVR to measure receptivity and reactivity to daily monitoring and six months post-baseline.

RESULTS Mean compliance rate was $70.6 \%$, with a morning rate of $72.4 \%$ and an evening compliance rate of $68.9 \%$ out of all possible surveys. IVR assessments of drinking and smoking were significantly associated with baseline paper-pencil reports of the same. African-American participants and those who reported more daily stressful events were more compliant. Between the baseline session and the 6-month follow-up, $68 \%$ of the sample reported engaging in some form of smoking behavior change ( $50 \%$ reduction in CPD, a quit attempt, pastmonth continuous abstinence). Nearly $80 \%$ reported increased awareness of their behavior due to the IVR and 40\% reported intentional behavior change from IVR monitoring. The odds of making a quit attempt at the 6-month follow-up were significantly higher among respondents who reported making purposeful changes to their smoking as a result of IVR monitoring $(\mathrm{AOR}=3.25, \mathrm{p}<0.05)$. CONCLUSIONS Reactivity was associated with behavior change outcomes. IVR may be a useful tool for motivating behavior change in smokers with alcohol-use problems.
\end{abstract}

\section{INTRODUCTION}

Interactive Voice Response (IVR) technology has become an increasingly popular and valid method for collecting Ecological Momentary Assessment (EMA) data on a variety of health-risk behaviors ${ }^{1-3}$, including daily alcohol use and cigarette smoking ${ }^{4-6}$. IVR data are recorded by having participants push

\begin{abstract}
AFFILIATION
1 Battelle Memorial Institute,

Arlington, United States

2 Hoda Elmasry was at

the Schroeder Institute

for Tobacco Research and

Policies Studies, Washington,

United States at the time of

data analysis and when the

manuscript was submitted

3 Old Dominion University,

Norfolk, United States

CORRESPONDENCE TO

Amy M. Cohn. Battelle

Memorial Institute, Arlington,

United States.

Email: cohn@battelle.org

\section{KEYWORDS}

interactive voice response, drinking, smoking, reactivity, compliance, behavior change
\end{abstract}

Received: 26 May 2017 Revised: 1 February 2018

Accepted: 2 February 2018 the buttons on the keypad of their telephone to answer a set of pre-recorded survey questions. IVR offers several advantages over paper-and-pencil data collection methods ${ }^{7}$. It provides respondents with a level of privacy in reporting sensitive behavior; allows a shorter recall period that improves confidence of the causal sequences among factors ${ }^{8}$; and improves 
measurement of moderators and mediators of behavior by immediately time-stamping and recording data.

IVR offers several advantages over other forms of EMA, like web-based, email, text messaging or smartphone app data collection ${ }^{7,9}$. Other forms of EMA have a higher potential for breach of confidentiality than IVR. Data transmitted via email are not always secure because one cannot guarantee that data travel directly from point to point ${ }^{10}$. Further, communicating through a mobile device, such as with an app, can leave user's personal information vulnerable to attack by viruses and malware. Additional protection must be taken to secure transmission of data sent via mobile phone or email, which may be costly or require computer programming knowledge. Similarly, textmessaging data goes through the cellular provider before it is sent to the device at the research site and often contains at least one form of identifiable information (name, phone number) and one form of private or confidential research information ${ }^{10}$. With IVR, however, data are recorded and stored on a password protected database and accessed only by approved study personnel. Older individuals may be less familiar with text messaging or how to use an app, making use of these EMA tools more burdensome for certain age groups. Furthermore, IVR can be deployed with any touchtone phone, thus a smartphone is not required to use IVR. Studies using more advanced EMA technologies may screenout individuals who do not own smartphones, thereby reducing the generalizability of study findings.

The potential for missing data and lack of compliance with IVR monitoring are two limitations that can reduce statistical power and affect estimation bias ${ }^{11,12}$. Compliance to IVR protocols is important as it has been shown to positively affect treatment outcomes and improve treatment and medication adherence for a variety of health behaviors ${ }^{13-15}$. For studies with drinkers or smokers, compliance rates range significantly from $46 \%$ to over $90 \%^{11,15-18}$. Compliance may be related to the timing of assessments, the amount of time between assessments, the number of assessments per day, and the type of behaviors being assessed. Further, some respondents may have limited time on their cell phones to complete phone calls, others travel due to work or have varying access to a phone, others may be highly motivated to complete the surveys for the financial incentives, as well as for the personal growth. For alcohol use specifically, answers may be influenced by degree of intoxication at the time of the call. Assessment reactivity, or the prospect that respondents may change their behavior as a result of daily monitoring, may also result from IVR monitoring ${ }^{19}$, although results have been mixed. Several studies of daily diary reporting indicate that reactivity is unlikely, or if it does exist, does not significantly impact measurement validity ${ }^{7,20}$. However, a separate line of research suggests that IVR monitoring may be an effective tool for behavior change $^{13,14,21,22}$

Babor and Del Boca ${ }^{23}$ proposed a theoretical model that identifies the cognitive, social and psychological factors that may affect participant self-reports during the question-and-answer process, which could extend to IVR self-reporting of alcohol and smoking. Key parameters within this framework are respondent characteristics and motivational processes that may affect the use of and compliance with assessment instruments. Respondent characteristics refer to enduring qualities of the individual who is completing the assessment, such as personality traits or demographic factors. Motivational characteristics are those factors that could affect the extent to which a respondent conforms to and complies with the assessment instructions, including substance use severity and desire to change behavior.

Individuals with co-occurring substance use disorders, like smoking and alcohol use, may experience unique barriers in complying with the IVR, and may react to the IVR differently than those without comorbidity. First, individuals with more severe symptomatology may have difficulty in maintaining motivation to continuously engage with the IVR system. Three prior studies with samples of drinkers showed that IVR compliance was inversely related to alcohol problem severity ${ }^{24,25}$. It is possible that respondents with concurrent nicotine dependence and alcohol-use problems may have lower IVR compliance than those reported in the current literature, which has focused on either smokers or drinkers, as separate groups ${ }^{5,12,24,25}$; none has focused on smokers who are also risky drinkers. Second, concerns have been raised about whether cognitive, emotional or physical impairments associated with substance-use behavior may interfere with one's ability to complete IVR surveys ${ }^{26}$. Numerous studies in the alcohol-treatment literature show a strong 
correlation between chronic and heavy drinking with neuropsychological impairment, including problems with attention and working memory ${ }^{27}$. Smoking has also been cited as a risk factor for dementia and decline in cognitive abilities ${ }^{28}$, factors that could interfere with reporting behavior, particularly in smokers who consume a pack or more of cigarettes a day. Third, individuals with more severe problems may also have limited funds to pay for the minutes accrued by each IVR survey, thus reducing their overall adherence to the survey schedule. One recent study showed that drinkers with greater financial stability were more compliant with the IVR system ${ }^{24}$, suggesting that the financial status of the respondent may be an important indicator of IVR utilization. Fourth, individuals who smoke and drink may also experience a wide array of psychosocial stressors (occupational distress, health or financial problems), which could interfere with motivation, with their ability to answer calls promptly, or to complete surveys in the allotted time frame. Individuals with more severe substanceuse problems may want to minimize the extent of their use behavior and could be reluctant to engage in self-monitoring protocols if they are less motivated to change. They could be concerned that daily tracking would reveal daily, and perhaps harmful patterns of use they may wish to ignore or to overlook. Finally, those who are highly motivated to change their substance use may find frequent or daily monitoring particularly useful, as it may increase awareness of their use patterns and provide insight into ways to alter these patterns.

More research is needed to evaluate the parameters of IVR compliance and reactivity in respondents who may have greater problem severity than samples previously examined in published IVR studies. No study, to our knowledge, has examined factors associated with IVR compliance in a comorbid group of smokers who drink at risky levels. The lack of published literature in this area is noteworthy, because the co-use of alcohol and tobacco is more prevalent among US adults than use of either substance alone ${ }^{29}$. To fill this knowledge gap, the first aim of this study was to examine the prevalence and correlates of IVR compliance in a sample of smokers with comorbid alcohol use disorders (AUDs) and alcohol-related problems, specifically to determine if IVR compliance varies with substance-use severity. Based on findings from prior studies, we hypothesized that age, income and motivation to change drinking and smoking would be positively associated with compliance; while greater alcohol consumption, nicotine dependence severity, negative daily life events, and the desire to use cigarettes or alcohol to cope with problems would be inversely associated with IVR compliance. Given that IVR monitoring can increase awareness of behavior and promote behavior change, the second aim was to examine the receptivity and self-reported reactivity to IVR (i.e. behavior change in response to IVR monitoring), as well as the impact of reactivity on smoking behavior change outcomes at a 6-month follow-up. While one of the pitfalls of EMA may be distortions or inaccuracies of data collection due to unintentional behavior change from self-monitoring, this may also be a strength of EMA. Individuals may be able to make changes to their behavior, or improve their motivation to change, through low-cost and broad-reaching self-monitoring protocols.

\section{METHODS}

\section{Participants}

Data were collected in a large Northeastern city in the United States between 2013 and 2015 by trained Masters' level study personnel. Participants were 84 risky drinking smokers recruited via print and web-based advertisements that asked for 'smokers who are regular drinkers'. Inclusion criteria were: 1 ) age 18-65 years, 2) smoked > 10 cigarettes/day, 3) consumed alcohol at NIAAA-defined risky drinking levels [> 2 drinks/day and 14 drinks/week for men; $>1$ drink/day and $>7$ drinks/week for women $]^{30}$, and 4) reported a desire to quit smoking within the next 6 months. Exclusion criteria were: 1) severe psychiatric disturbance, 2) potential for significant alcohol withdrawal, 3) use of cocaine, heroin or crack, 6 out of 7 days a week in the past month $\left.{ }^{\mathrm{a}}, 4\right)$ or pregnant

a Given human subjects concerns, we excluded participants with active suicidal or homicidal features, or whose stimulant or opiate use represented an immediate health hazard, because the proposed study design did not address or treat these symptoms and had the potential to place the participant at greater risk for medical and psychological problems relative to the benefits of participation. Because the primary aim of this study was to examine the longitudinal behavior change outcomes of untreated problem drinking smokers, those who provided evidence of these behaviors at the time of the screening interview were given referrals for treatment, but were no longer considered eligible for the study. We considered the impact of excluding these people on the overall science of the project, but felt it was more important to provide referrals for treatment than to observe these individuals potentially decline or decompensate in their mental health or substance use over the course of the study without intervening. 
or planning to become pregnant. Using criteria that were based on the clinical practice guidelines for treating tobacco dependence ${ }^{31}$, desire to quit smoking in the next 6 months was measured on a five-point scale, with response options: 1) yes, definitely, 2) yes, probably, 3) unsure, 4) no, I would rather not, or 5) no, definitely not. Individuals who answered no, I would rather not, or no, definitely not were not eligible. Inclusion/exclusion criteria were assessed via self-report and semi-structured interview. This study was approved by the Schulman IRB, protocol \# 201304003. All data were collected in accordance with the Declaration of Helsinki.

\section{Baseline measures}

Measures listed below are standard instruments commonly used in smoking and/or alcohol research studies and are psychometrically valid and reliable. After providing informed consent, demographic information, including gender, race, ethnicity, income and employment were collected. Readiness to quit smoking was assessed using the Contemplation Ladder (CL), a self-report instrument that measures readiness to quit smoking on a 10-point Likert-type scale ('no thoughts of quitting' to 'taking action to quit' $)^{32}$. The CL has shown good convergent validity with other measures of motivation to change, and predicts longer term readiness to quit smoking in samples of adults ${ }^{32-34}$. The Stages of Change and Treatment Readiness Scale (SOCRATES) ${ }^{35}$ is a 19item self-report instrument that was used to assess motivation to change drinking behavior (1 - strongly disagree to 5 - strongly agree) and contains three subscales: Ambivalence, Recognition, and Taking Steps. The Smoking Temptations Inventory (STI) ${ }^{36}$ is a 9-item validated self-report measure that was used to assess temptation to smoke in nine highrisk smoking situations ( 1 - not at all tempted to 5 - extremely tempted). The STI has demonstrated good reliability and internal consistency in samples of adult smokers ${ }^{36}$, with a total score reflecting overall temptation to smoke. The SOCRATES demonstrates excellent test-retest reliability in drinkers and good convergent validity with measures of longer term drinking ${ }^{35}$. Nicotine dependence was measured using the 6-item self-report Fagerström Test for Nicotine Dependence (FTND) ${ }^{37}$, which has demonstrated high reliability and validity, and good internal consistency in samples of daily smokers. The Structured Clinical Interview for DSM-IV (SCID) ${ }^{38}$ is a semi-structured interview that was used to assess lifetime and current alcohol use disorder (AUD), as well as number of lifetime AUD symptoms. The SCID was administered by Master's level trained interviewers and all SCIDs were reviewed by the PI of the study. The Time Line Follow Back interview (TLFB ) ${ }^{39}$ is an interviewer administered questionnaire that was used to assess day-level drinking and smoking behavior within the 90 days prior to the baseline assessment. The TLFB yielded the following indices that were used in the current analyses: mean drinks per drinking day (MDDD), proportion heavy drinking days (6+ drinks/day, PHDD), and cigarettes per smoking day (CPD) within the 90 days prior to the baseline session. Our threshold for PHDD aligned with criteria from the Alcohol Use Disorder Identification Test ${ }^{40}$. The TLFB has shown high test-retest reliability, and strong correlations between participant and collateral reports of drinking and smoking ${ }^{39,41}$.

\section{IVR assessments}

At the baseline session, participants completed a 20-minute IVR training, wherein they were taught to record drinking data (in standard drinks) and completed a practice IVR survey from their telephone. Participants were instructed to record their responses to pre-recorded survey questions by pushing buttons on the keypad of their telephone.

Participants recorded daily factors in the following areas: 1) quantity and frequency of beer, wine and liquor consumed in standard drink conversions since the previous assessment; 2 ) cigarettes consumed since the previous assessment; 3 ) degree of temptation to smoke in nine high-risk situations since the previous assessment (items adapted from the STI; 1 - not at all tempted to 5 - extremely tempted $)^{36}$; 4) smoking cigarettes to cope with any negative daily events since the previous assessment (yes/no); 5) drinking alcohol to cope with any negative daily events since the previous assessment (yes/no); and 6) the occurrence of specific life events since the previous assessment (argument with a friend or family member, a financial problem, a work-related problem, and a problem related to their health or well-being issue). Other factors known to correlate with drinking and smoking, including mood and craving, were also included in the 
daily assessments but are not reported in this paper. IVR assessment items were selected to parallel the constructs measured at baseline (e.g. self-efficacy) and have established psychometric properties ${ }^{2,42,43}$.

For 28 days following the baseline, participants recorded daily alcohol use, smoking, smoking temptation and smoking-related risk factors. Prompts (e.g. calls to their telephone) were programmed electronically to occur twice a day at random times within each of two 4-hour periods, one corresponding to the morning and one the evening. The hour of administration for the morning and evening surveys differed for each participant, as prompts were programmed to coincide with the respondents' sleepwake cycle (i.e. the usual times they wake up and go to sleep), which was collected at the baseline survey. The IVR system was configured to call (prompt) the participant's cell phone and was enabled so that participants could directly access the survey after they received the call by pressing ' 1 ' on their phone keypad. Prompting lasted for 10 seconds, and a participant had 2 minutes to respond. If the prompt was missed, the IVR system cued two additional prompts, each 5 minutes apart. Because data were time-stamped, we recorded whether surveys were completed outside of the scheduled sleep-wake cycle. The IVR system was set up so that no random prompts were issued within 2 hours of each other, and separate morning and evening interviews were programmed to facilitate different questions at each time period. IVR interviews were date- and timestamped and recorded immediately. Several system features were used to promote adherence, including clear prompts, minimal skip outs, and ability to return to questions.

To enhance IVR compliance, participants received $\$ 15$ each week they participated in the IVR phase (total of \$60). Additional bonus incentives were provided at $\$ 0.50$ for each completed call; as well as $\$ 2$ per week for completing prompts 6 out of 7 days, or $\$ 5$ per week for completing prompts for all 7 days. Thus, participants were eligible to receive up to $\$ 108$ if they completed all IVR interviews.

\section{Post-IVR survey}

At the end of IVR monitoring (1-month postbaseline), participants completed a questionnaire to measure receptivity and reactivity to IVR. The post-
IVR survey was developed specifically for this research study, in collaboration with several experts in the field of EMA data collection. To measure receptivity, participants were asked: 'Did you feel the monitoring schedule was burdensome or took too much time?', and 'Were the questions easy to understand?'. All items used the same 5-point scale (0 - Not at all, 4 Extremely). To measure reactivity, participants were asked: 'To what extent did you feel that the daily phone calls may have caused you to be more aware of your behavior?' (0 - Not at all, 4 - Extremely), and 'Did you begin to notice any behaviors more than before, and if so which ones? (select all that apply)', with response options including smoking and alcohol use (yes/no). Participants were also asked: 'Did you find that you purposely started to make changes to your behavior because of the daily monitoring?' ( 0 - Not at all, 4 - Extremely) and 'which behaviors did you purposely make changes to? (select all that apply)', with response options for smoking and alcohol use (yes/no).

In total, $85 \%$ of the sample $(n=72)$ completed the post-IVR survey. Non-completers had a higher income $[\mathrm{F}(1,74)=4.79, \mathrm{p}<0.05]$, and were more likely to be male $\left(\mathrm{x}^{2}=4.99, \mathrm{p}<0.05\right)$.

\section{Six-month follow-up}

A follow-up assessment of alcohol and smoking behavior change was completed 6 -months postbaseline and included re-administration of the TLFB to obtain measurements of smoking and alcohol use behavior, as well as measurements of 7-day and 30day point prevalence abstinence from smoking. Those who reported not smoking for at least 24 hours were considered having made a quit attempt.

In total, $84 \%$ completed the 6-month follow-up $(n=71)$. Those who completed the 6-month survey were more likely to be African-American $\left(x^{2}=7.39\right.$, $\mathrm{p}<0.05)$.

\section{Analytic plan}

We assessed two compliance rates, one for percentage of surveys answered over the course of the study, and the other for percentage of survey questions answered over the course of the study. The overall compliance rate for surveys was based on the number of surveys in which at least one question in that survey was answered, divided by the total number of surveys 
possible. The denominator was the total number of morning and evening surveys multiplied by the total number of participants ([28 morning surveys +28 evening surveys]x77 participants $=4312$, the total number of surveys in the study). Given the study design (separate morning and evening surveys), overall compliance rate was also stratified by time of day, yielding compliance rates overall, as well as in the morning and in the evening. Weekend vs weekday compliance rates, as well as average survey response times were also examined. Question-level compliance rate was the number of all questions answered across all study days and participants, divided by the total number of possible questions that could be answered across all study days and participants. Because there were 47 total possible questions in the morning survey, 40 total possible questions in the evening survey, 28 study days and 77 respondents, the denominator for this analysis was equal to $187572([47+40] \times 28 \times 77)$.

Descriptive statistics examined differences in compliance rates and missed surveys overall, based on daytime-evening and weekday-weekend reports. Correlation coefficients were then obtained to examine the association between IVR-assessed measurements of smoking, drinking and smoking temptation with corresponding baseline paperpencil reports. Next, linear regression models were performed to examine the association of demographic, tobacco and alcohol use factors, motivation to change, and daily life events (i.e. argument with a family or friend, financial problems, health problems) with compliance rates, to determine if compliance rates varied as a function of greater problem severity, motivation to quit smoking, and stressful life events.

Prevalence, correlates and predictive validity of IVR receptivity and reactivity were then examined. Regression models (binary logistic or linear regression, depending on the outcome) examined the predictive utility of IVR reactivity on change in alcohol and smoking behavior from baseline to 6-months. The independent variables for these models were increased awareness of smoking (or drinking) and purposeful changes to smoking (or drinking) resulting from IVR self-monitoring. Change in smoking behavior from baseline to 6-months was examined in several different ways. First, using linear regression analysis, we examined changes in cigarettes/day and nicotine dependence severity (as separate outcomes), controlling for baseline reports of the outcome, motivation to quit smoking, and IVR compliance (\% of surveys completed). Second, a variable was created to examine overall smoking behavior change, based on reports of at least one of the following (dichotomous, yes/no): 1) a $50 \%$ reduction in cigarettes smoked per day from baseline to the 6 -month follow-up; 2) cessation attempt lasting $>1$ day and $<7$ days; or 3) past-month continuous abstinence from smoking. This broad definition of smoking behavior change was chosen to include a variety of different change outcomes, similar to another study ${ }^{44}$. Each outcome was also examined in separate models, with the exception of past-month continuous abstinence, due to small cell size $(n=1)$. Because this outcome was dichotomous, bivariate logistic regression analyses were employed, using the independent variables capturing increased awareness of smoking and purposeful changes to smoking.

For changes in alcohol use behavior, linear regression analyses were used to examine changes in percentage heavy drinking days and mean drinks per drinking days from baseline to 6-months, controlling for baseline reports of the outcome, motivation to quit smoking, and IVR compliance.

\section{RESULTS}

\section{Participant characteristics}

Table 1 shows participant characteristics of the 77 respondents who took at least one IVR survey. Participants were middle aged adults $(M=45.8$, $\mathrm{SD}=10.8$ ), unmarried (84\%), African-American $(86 \%)$, and male $(52 \%)$. Nearly all participants had either a lifetime AUD (93.5\%) or a past-month AUD (64.9\%). Participants smoked 13.8 cigarettes/day $(\mathrm{SD}=7.4)$, reported a moderate degree of nicotine dependence $(\mathrm{M}=6.4)$, and consumed 7.4 drinks ( $\mathrm{SD}=5.6)$ per drinking day at baseline. Even though they were heavy drinkers, most (90\%) indicated little recognition of the need to make changes to their drinking and $89.6 \%$ was not actively taking steps to change their drinking.

\section{Compliance rates, missed reports and survey completion time}

Table 2 shows overall compliance rates and missed reports by time-of-day and by weekend vs weekday. Participants completed an average of 39.56 out of 56 
Table 1. Participant characteristics $\mathbf{n}=77$ respondents

$\begin{array}{lll}\text { Demographic factors } & & \\ & \text { Mean } & \text { SD } \\ \text { Age } & 45.6 & 10.8 \\ & \mathrm{~N} & \% \\ \text { Gender } & & \\ \text { Female } & 37 & 48.1 \\ \text { Male } & 40 & 52.0 \\ \text { Race } & & \\ \text { White } & 7 & 9.1 \\ \text { African American } & 66 & 85.7 \\ \text { Other } & 4 & 5.2 \\ \text { Employed } & 39 & 50.6 \\ \text { Not employed } & 38 & 49.4 \\ \text { Baseline alcohol and tobacco use factors }\end{array}$

Table 2. Compliance rates across morning and evening, weekday and weekend, surveys out of $n=$ 4312 total possible surveys and missed surveys

$\begin{array}{lll}\text { Survey compliance }\left(n_{\text {max }}\right. & \text { (13/2) } & \\ & \mathrm{N} & \% \\ \text { Overall surveys } & 3046 & 70.6 \\ \begin{array}{l}\text { Study days with either } \\ \text { morning or evening } \\ \text { completed surveys }\end{array} & 1707 & 79.2 \\ \begin{array}{l}\text { Morning vs Evening } \\ \text { Morning }\end{array} & \\ \begin{array}{l}\text { Evening } \\ \text { Weekend vs Weekday }\end{array} & 1560 & \\ \text { Weekend } & 840 & 68.4 \\ \text { Weekday } & 2206 & 68.1 \\ \end{array}$

Participants will missing surveys (n 77)

$\begin{array}{lll}\text { Overall } & \text { N } & \% \\ \text { Missed 0-5 surveys } & 28 & 36.4 \\ \text { Missed 6-10 surveys } & 9 & 11.7 \\ \text { Missed 11+ surveys } & 40 & 51.9 \\ \text { Morning Surveys } & & \\ \text { Missed 0-5 surveys } & 44 & 57.1 \\ \text { Missed 6-10 surveys } & 11 & 14.3 \\ \text { Missed 11+ surveys } & 22 & 28.6 \\ \text { Evening Surveys } & & \\ \text { Missed 0-5 surveys } & 40 & 13.0 \\ \text { Missed 6-10 surveys } & 10 & 35.1 \\ \text { Missed 11+ surveys } & 27 & \end{array}$

total possible surveys ( $\mathrm{n}=3046$ total surveys), yielding an average compliance rate of $70.6 \%(\mathrm{SD}=29.79$, range: $1.79-100$ ), with a morning rate of $72.4 \%$ and an evening compliance rate of $68.9 \%$ out of all possible surveys that could have been completed for those periods. There were significantly higher compliance rates in the mornings compared to the evenings ( $\mathrm{t}$ : $2.48, \mathrm{p}<0.01)$ and on weekdays $(71.7 \%)$ vs weekends $(68.1 \%)(\mathrm{t}: 2.35, \mathrm{p}<0.05)$. A third of the sample (34\%) completed all 28 IVR days, only 3 participants completed just one day. Initial compliance rate was $75.7 \%$ in week 1 and fell to $65.9 \%$ by week 4 .

In terms of missed reports aggregated across both morning and evening surveys, $36 \%$ missed up to five of 
the 56 possible surveys; $11.7 \%$ missed between 6 and 10 surveys; and half missed 11 or more surveys. The breakdown between morning and evening surveys was slightly different, with the majority missing up to 5 surveys for both morning and evening; a quarter of the sample missed 11 or more morning surveys; and over a third (35\%) missed 11 or more evening surveys.

Survey completion time was 5.6 minutes $(\mathrm{SD}=2.2)$ and decreased significantly over the course of the study $(\beta:-2.5, p<0.0001)$. One third of evening surveys $(33.9 \%)$ and slightly fewer $(19 \%)$ of the morning surveys were taken outside of the predefined sleepwake cycle collected at baseline. The compliance for all survey questions taken was $67.4 \%(\mathrm{SD}=41.2)$.

\section{Association between IVR and paper-pencil reports}

Aggregated daily responses of smoking temptation from the IVR-administered STI were significantly and positively correlated with baseline paperpencil administered STI scores $(\mathrm{r}=0.53, \mathrm{p}<0.001)$. Aggregated daily responses of smoking $(r=0.27$, $\mathrm{p}=0.02)$ and drinking $(\mathrm{r}=0.61, \mathrm{p}<0.001)$ were also significantly correlated with corresponding baseline TLFB scores. Cronbach alpha for the 9-item IVRadministered STI was high $(a=0.94)$ for both the morning and evening assessments, which was slightly higher than the value $(a=0.86)$ for the baseline paperpencil administration of the STI.

\section{Baseline and daily correlates of IVR compliance}

Table 3 shows results of bivariate linear regression models of demographic and baseline correlates of IVR compliance (continuous outcome), with variables entered in the model individually. Compliance rates among White participants $(\mathrm{M}=49.5 \pm 23.7)$ were significantly lower than African-American participants $(\mathrm{M}=74.2 \pm 28.7, \mathrm{p}=0.03)$. No other significant baseline and demographic correlates of IVR compliance emerged ${ }^{\mathrm{b}}$.

Table 4 shows results of linear regression models examining the association between aggregated daily events with overall compliance rates. We conducted these analyses because we proposed that respondents experiencing more daily hassles and stressful life
Table 3. Bivariate regressions of demographic and baseline correlates of IVR survey compliance

Survey compliance rate

Demographic factors

Gender

Female

3.28

6.83

0.63

Male (ref)

$\begin{array}{llll}\text { Age } & 0.15 & 0.32 & 0.64\end{array}$

Race

White

$-24.67 \quad 11.48$

0.03

African American (ref)

Other

$-24.61 \quad 14.87$

Employment status

Employed

Not employed (ref)

Baseline factors

Temptation to smoke

$\begin{array}{lll}0.66 & 0.46 \quad 0.15\end{array}$

Mean drinks/drinking day

$\begin{array}{lll}-0.33 & 0.61 & 0.59\end{array}$

Proportion heavy drinking days

Cigarettes per day

$\begin{array}{lll}0.48 & 0.46 \quad 0.30\end{array}$

Nicotine dependence

$\begin{array}{lll}1.43 & 1.53 & 0.35\end{array}$

Readiness to quit smoking

$1.27 \quad 1.43$

Motivation to change drinking (SOCRATES)

Actively taking steps to change drinking

High 14.8

Low (ref)

Ambivalence

High $-0.42 \quad 6.91$

Low (ref)

Recognition of need to change drinking

High $-19.9$

Low (ref)

Alcohol use disorder (AUD)

Lifetime $-5.31$

Current (ref)

No

$5.71 \quad 14.09$

0.69 
Table 4. Association between aggregated daily events (over 28 days) and overall compliance rate

$\begin{array}{lccccc}\text { Darily event } & \text { Vean } & \text { SD } & B & \mathbb{R}^{2} & \boldsymbol{P} \\ \text { Argument with family } & 7.30 & 9.59 & 0.94 & 0.09 & 0.01 \\ \text { Work problem } & 2.84 & 6.87 & 0.75 & 0.03 & 0.13 \\ \text { Argument with friend } & 6.38 & 10.49 & 0.76 & 0.07 & 0.02 \\ \text { Financial problems } & 11.79 & 15.82 & 0.56 & 0.09 & 0.01 \\ \text { Health problems } & 5.73 & 11.76 & 0.41 & 0.03 & 0.16 \\ \text { Drinking to cope } & 10.33 & 14.05 & 0.63 & 0.09 & 0.01 \\ \text { Smoking to cope } & 15.99 & 17.49 & 0.59 & 0.14 & 0.001\end{array}$

Mean indicates average number of times participants reported the event occurring over the 28-day EMA monitoring period, aggregated across all respondents.

events may be less compliant with the IVR system. There were significant positive associations between arguments with family or friends, financial problems, drinking and smoking to cope, with overall compliance rate $($ all $\mathrm{p}<0.05)$.

\section{Prevalence, correlates and predictive utility of IVR receptivity and reactivity}

Figure 1 shows the proportion of respondents who provided receptivity ratings for the IVR monitoring protocol. The majority (70\%) reported that the IVR was 'not at all' or 'slightly' burdensome, and 'very much' or 'extremely' easy to understand ( $83 \%)$.

Figure 2 shows the proportion of respondents who reported increased awareness of their behavior due to IVR monitoring and purposeful change to their behavior from IVR. Most participants (80\%) reported that IVR monitoring caused them to be 'very much' or 'extremely' more aware of their behavior in general; $77 \%$ reported greater awareness of their cigarette smoking; and $66 \%$ reported greater awareness of their drinking. In total, $56 \%$ of respondents reported making intentional changes to their drinking, and $54 \%$ reported making intentional changes to their smoking. IVR compliance (\% of reports completed out of the total) was unrelated to intentional change to smoking or drinking, or to increased awareness of these behaviors.

Between the baseline session and the 6-month follow-up, $68 \%$ of the sample reported engaging in some form of smoking behavior change ( $50 \%$ reduction in CPD, a quit attempt, past-month continuous abstinence); with $40 \%$ making an intentional quit attempt, 35\% showing a 50\% reduction in cigarettes/
Figure 1. Proportion of respondents reporting that IVR monitoring was burdensome and easy to understand

Did you feel the monitoring schedule was burdensome or took too much time?

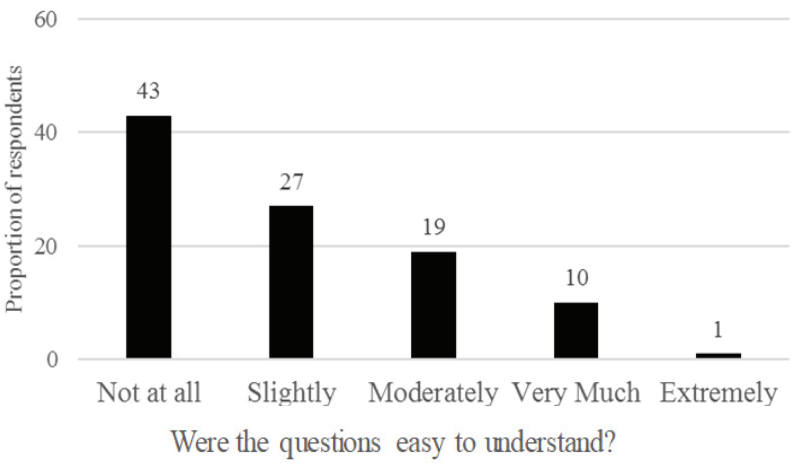

60

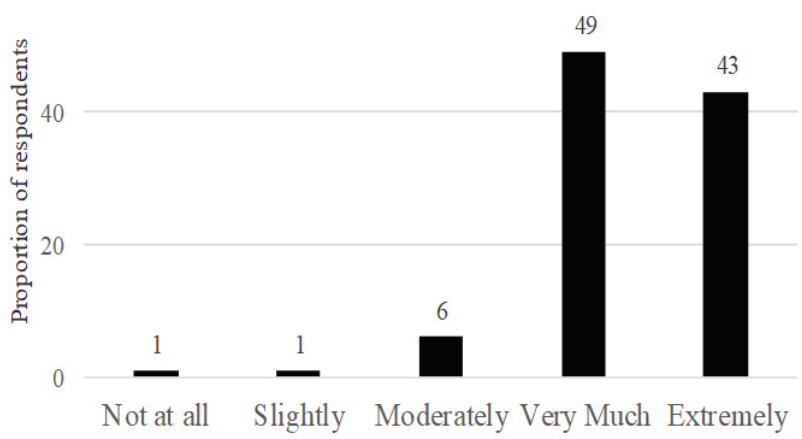

Figure 2. Proportion of respondents reporting increased awareness and purposeful behavior change from IVR monitoring

Increased awareness and purposeful change to behavior from IVR monitoring

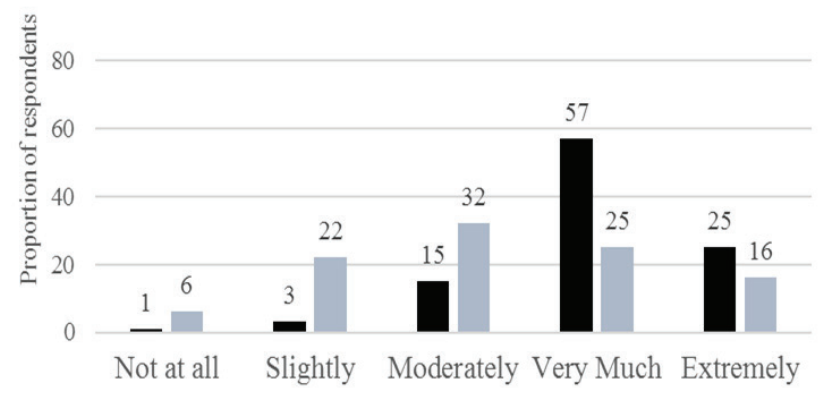

Increased awareness and purposeful change to smoking and drinking from IVR monitoring

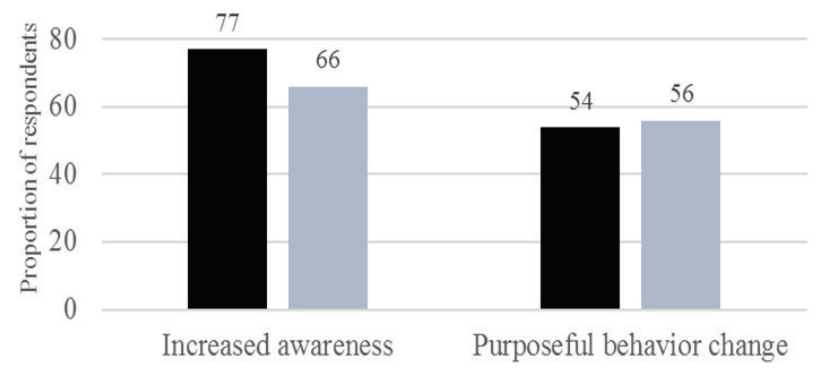


day, and one person reporting past-month continuous abstinence. Results from adjusted logistic regression models showed that the odds of engaging in some form of smoking behavior change at the 6-month follow-up were nine times larger among respondents who reported increased awareness of their smoking compared to those who did not $(\mathrm{AOR}=9.53, \mathrm{p}<0.01)$, even after controlling for baseline motivation to quit smoking and IVR compliance. The odds of making a quit attempt at the 6-month follow-up were three times greater among respondents who reported making purposeful changes to their smoking, as a result of the IVR, compared to those who did not $(\mathrm{AOR}=3.25, \mathrm{p}<0.05)$. There was a trend for those who reported increased awareness of their behaviors (overall) to engage in some form of smoking behavior change $(\mathrm{AOR}=1.56, \mathrm{p}=0.08)$, to make a smoking quit attempt $(\mathrm{AOR}=2.06, \mathrm{p}=0.05)$, and to make reductions in their percentage of heavy drinking days $(B=-0.06$, $\mathrm{p}=0.10$ ). No other significant effects of IVR reactivity were found.

\section{DISCUSSION}

This is the first study to evaluate the use of and receptivity to daily IVR assessments in a sample of untreated risky drinking smokers who were motivated to quit smoking. Most other EMA studies have included treated samples of drinkers and/or smokers, but these do not capture the naturally occurring changes in smoking and alcohol-related factors in untreated samples without the influence of intervention. Calls were completed on nearly $80 \%$ of days, with an overall compliance rate of $70 \%$, and individuals were more likely to comply with the survey in the mornings and on weekdays. Surveys lasted approximately 5 minutes, but survey completion time significantly decreased over time. Participants appeared to be accurately reporting their substanceuse behavior over the course of 28 days, as evidenced by significant and positive associations between IVR-assessed cigarette and alcohol use, and smoking temptation with corresponding baseline paper-pencil reports. Cronbach's alpha for the IVR-administered smoking temptation questionnaire was also high, providing further evidence that participants were not haphazardly responding to survey items. There were no obvious demographic or substance use factors, aside from race, that negatively affected compliance rates and that could have identified participants, at the outset of the study, in need of additional assistance and support utilizing the IVR system. Motivation to quit smoking could have been unrelated to IVR compliance because individuals who were in the study already had a high desire to quit smoking. Further, it is possible that the association between White race and lower compliance was a spurious effect, perhaps due to unequal numbers of White and Black respondents in the sample, as tests of mean differences can be affected by unequal cell sizes ${ }^{45}$. Replication of our study findings are warranted to determine whether compliance is differentially affected by race or ethnicity and whether generalizable.

Surprisingly, individuals who reported greater interpersonal and financial problems, and reported using drinking and cigarette smoking as forms of coping, were more compliant with the system. It is possible that those who experience more negative life events and who use cigarettes or alcohol to cope with these problems may have been using the IVR as a form of treatment or emotional support. This notion is consistent with other work showing that drinkers with more severe problems and consequences of use will make changes to their drinking, perhaps because they recognize the need to do so or because they are compelled to do so by family, friends or occupational circumstances ${ }^{46}$. The low-cost, convenience and confidentiality of IVR may make it a more acceptable method for behavior change than formal face-to-face treatment. It is also important to note that those who experienced financial problems had higher compliance rates. It is possible that because participants were compensated for their survey compliance, those with greater financial problems were more likely to be compliant in an effort to be paid more. Regardless, this finding suggests that contingency management and incentive-based interventions, which provide rewards for abstinence and behavior change, may be effective at enhancing compliance rates to a variety of treatment regimens in this group of smokers, including medication adherence, treatment session adherence, etc.

Participants overwhelmingly rated the IVR as easy to understand and to complete. This should allay some concerns that IVR surveys are burdensome and could potentially cause reporting fatigue. The majority of participants reported increased awareness of their 
drinking behavior and made intentional changes to this behavior as a result of IVR monitoring, but they did so regardless of the number of times they used the system. Further, the odds of making a quit attempt were significantly greater among those who reported increased awareness of their smoking behavior and intentional changes to their smoking post-IVR. This finding is somewhat inconsistent with that of Hufford et al. ${ }^{20}$ who showed minimal impact of EMA recording on behavior change, but their sample differed from ours in that they monitored college students who were not heavy drinkers or regular smokers, the monitoring period in their study was much shorter ( 2 weeks), and also our sample was motivated to make changes. While our findings suggest that IVR may be useful at increasing awareness of problematic substance-use behavior, this enhanced awareness does not appear to translate into robust behavior change, as no other indices of self-reported reactivity predicted alcohol or cigarette smoking behavior change. Further, it is important to note that respondents said that IVR helped stimulate behavior change, although there was no significant association between compliance rates and these self-reported measurements of reactivity. It is possible that respondents are unaware of why they made changes, or that even a small amount of IVR monitoring can have an impact on changing attitudes and behavior. We cannot rule out whether the link between reactivity and subsequent behavior change was due to natural shifts in behavior over time, as respondents who self-selected into the study were already highly motivated to quit smoking. It is important to note that respondents were told during informed consent that the purpose of the study was to examine associations of daily smoking and drinking patterns on subsequent cessation behavior. It is possible that this information could have affected IVR reactivity. Similar to Hufford et al. ${ }^{20}$, we did not have a non-EMA control group to experimentally compare change outcomes. Taken together, findings suggest that special populations are receptive to IVR monitoring, and that IVR may help motivated smokers to make positive behavior change. The extent to which daily monitoring can be supplemented with more intensive forms of treatment (psychotherapy, pharmacotherapy) to enhance motivation to change and longer term change outcomes, should be examined.
The findings of this study demonstrate that using IVR to collect daily smoking and drinking behavior in smokers with co-occurring alcohol use problems is feasible and provides psychometrically valid data. We have shown that attitudinal and behavioral change in response to IVR monitoring is possible in a sample that is not likely to seek formal treatment. This has important implications for the use of low cost, broadreaching assessments or brief interventions with subgroups who have substance-use comorbidities. We are unaware of any study that has directly examined changes in attitudes or behavior following IVR monitoring and the impact of such self-reported changes on subsequent natural change outcomes in a sample of risky drinking smokers who are not seeking treatment. This approach allowed us to capture the potential impact of daily self-monitoring on naturally occurring changes in smoking and alcohol use, without the influence of counseling or medication. While there was convergence across IVR-administered measures of smoking and drinking with corresponding paperpencil reports, the strength of associations was not large ${ }^{47}$. This further highlights that daily assessments provide qualitatively different information than what can be measured via traditional paper-and-pencil questionnaires ${ }^{8}$.

There are several limitations of this study. First, retrospective reports of drinking and smoking were not collected at the end of the IVR to crossvalidate behavior over the 28-day reporting period. Other studies have taken such an approach and found high levels of correspondence between IVR and retrospective measurements of smoking and drinking ${ }^{5,48}$. Second, most participants in the current study were African-American and findings may not be generalizable to other groups of heavy drinking smokers. Rates of current smoking are almost three and a half times higher among Black than White adults in the US ${ }^{49}$, making this an important target population for future research. Third, data are correlational in nature and causal conclusions cannot be made. We cannot determine whether daily stressful events predict compliance rates, or whether degree of compliance somehow affects the intensity and number of stressful events reported, perhaps through increased awareness of these events. Because we used aggregated assessments of daily stressful events, we did not control for differences 
across participants with respect to the number of reports they provided. Fourth, we are unable to determine whether self-monitoring had an impact on changes in attitudes and behaviors, beyond no selfmonitoring. Fifth, intoxication at the time of the IVR assessment was not queried. However, daily reports with drinkers indicate that participants can reliably and accurately report on the IVR with a high degree of detail even when intoxicated ${ }^{4,50,51}$. Finally, several other IVR studies have used similar, if not smaller, samples when reporting compliance rates of alcohol, substance use, or other behaviors, thus allaying potential concerns about the study sample size.

\section{CONCLUSIONS}

Our findings support the feasibility of using IVR with smokers who are heavy drinkers and show that prestudy risk factors (i.e. alcohol use problem severity, nicotine dependence) do not affect the degree to which these individuals are likely to comply with the IVR system. Further, IVR appeared to help stimulate and enhance the process of positive behavior change in a group motivated to make changes. Identifying barriers to implementing and executing IVR with high-risk populations could inform modifications to current and ongoing EMA protocols to improve efficiency and reduce participant burden. Further, isolating factors that can influence utilization and reactivity to IVR monitoring can lead to the provision of improved guidelines regarding the use of IVR as a brief screening and intervention tool.

\section{REFERENCES}

1. Corkrey R, Parkinson L. Interactive voice response: review of studies 1989-2000. Behav Res Methods Instrum Comput. 2002;34(3):342-353. doi:10.3758/bf03195462

2. Cranford JA, Tennen H, Zucker RA. Feasibility of using interactive voice response to monitor daily drinking, moods, and relationship processes on a daily basis in alcoholic couples. Alcoholism: Clinical and Experimental Research. 2010;34(3):499-508. doi:10.1111/j.1530-0277.2009.01115.x

3. Tucker JA, Simpson CA, Huang J, Roth DL, Stewart KE. Utility of an interactive voice response system to assess antiretroviral pharmacotherapy adherence among substance users living with HIV/AIDS in the rural South. AIDS Patient Care STDS. 2013;27(5):280-286. doi:10.1089/apc.2012.0322

4. Mundt J, Bohn MJ, King M, Hartley MT. Automating standard alcohol use assessment instruments via interactive voice response technology. Alcoholism: Clinical and Experimental Research. 2002;26(2):207211. doi:10.1097/00000374-200202000-00007

5. Toll BA, Cooney NL, McKee SA, O'malley SS. Do daily interactive voice response reports of smoking behavior correspond with retrospective reports? Psychol Addict Behav. 2005;19(3):291. doi:10.1037/0893-164x.19.3.291

6. Helzer JE, Badger GJ, Searles JS, Rose GL, Mongeon JA. Stress and alcohol consumption in heavily drinking men: 2 years of daily data using interactive voice response. Alcoholism: Clinical and Experimental Research. 2006;30(5):802-811. doi:10.1111/j.1530-0277.2006.00093.x

7. Shiffman S. Ecological momentary assessment (EMA) in studies of substance use. Psychol Assess. 2009;21(4):486. doi:10.1037/a0017074

8. Conner T, Tennen H, Fleeson W, Barrett L. Experience sampling methods: A modern idiographic approach to personality research. Soc Personal Psychol Compass. 2009;3(3):292-313.

doi:10.1111/j.1751-9004.2009.00170.x

9. Shiffman S, Hickcox M, Paty JA, Gnys M, Kassel JD, Richards TJ. Progression from a smoking lapse to relapse: Prediction from abstinence violation effects, nicotine dependence, and lapse characteristics. J Consult Clin Psychol. 1996;64(5):993. doi:10.1037/0022-006x.64.5.993

10. LuxSci. Is text messaging HIPAA compliant? http:// luxsci.com/blog/is-text-messaging-hipaa-compliant. html. Published, 2012. Accessed October 12, 2012.

11. Kaminer Y, Litt MD, Burke RH, Burleson JA. An interactive voiceresponse(IVR) systemforadolescentswithalcoholuse disorders: a pilot study. Am J Addict. 2006;15(sup1):122125. doi:10.1080/10550490601006121

12. Toll BA, Cooney NL, McKee SA, O’Malley SS. Correspondence between Interactive Voice Response (IVR) and Timeline Followback (TLFB) reports of drinking behavior. Addict Behav. 2006;31(4):726-731. doi:10.1016/j.addbeh.2005.05.044

13. Lindsay JA, Minard CG, Hudson S, Green CE, Schmitz JM. Using prize-based incentives to enhance daily interactive voice response (IVR) compliance: A feasibility study. J Subst Abuse Treat. 2014;46(1):74-77. doi:10.1016/j.jsat.2013.08.003

14. Stacy JN, Schwartz SM, Ershoff D, Shreve MS. Incorporating tailored interactive patient solutions using interactive voice response technology to improve statin adherence: results of a randomized clinical trial in a managed care setting. Population health management. 2009;12(5):241-254. doi:10.1089/pop.2008.0046

15. Schroder KE, Tucker JA, Simpson CA. Telephone-based self-change modules help stabilize early natural recovery in problem drinkers. Journal of studies on alcohol and drugs. 2013;74(6):902. doi:10.15288/jsad.2013.74.902 
16. Toll BA, Cooney JL, McKee SA, O’Malley SS, Cooney NL. Correspondence of interactive voice response (IVR) reports of nicotine withdrawal, craving, and negative mood with questionnaire ratings. Nicotine \& tobacco research. 2008;10(6):1057-1064. doi:10.1080/14622200802097498

17. Tucker JA, Foushee HR, Black BC. Behavioral economic analysis of natural resolution of drinking problems using IVR self-monitoring. Exp Clin Psychopharmacol. 2008;16(4):332. doi:10.1037/a0012834

18. Cohn A, Hagman BT, Moore K, Mitchell J, Ehlke S. Does negative affect mediate the relationship between daily PTSD symptoms and daily alcohol involvement in female rape victims? Evidence from 14 days of interactive voice response assessment. Psychol Addict Behav. 2014;28(1):114. doi:10.1037/a0035725

19. Barta W, Tennen H, Litt M. Measurement reactivity in diary research. Handbook of research methods for studying daily life. 2012:108-123.

20. Hufford M, Shields A, Shiffman S, Paty J, Balabanis M. Reactivity to ecological momentary assessment: An example using undergraduate problem drinkers. Psychol Addict Behav. 2002;16(3):205-211. doi:10.1037//0893-164x.16.3.205

21. Helzer JE, Rose GL, Badger GJ, Searles JS, Thomas $\mathrm{CS}$, Lindberg SA, et al. Using interactive voice response to enhance brief alcohol intervention in primary care settings. Journal of Studies on Alcohol and Drugs. 2008;69(2):251-258. doi:10.15288/jsad.2008.69.251

22. Rose GL, Skelly JM, Badger GJ, Naylor MR, Helzer JE. Interactive voice response for relapse prevention following cognitive-behavioral therapy for alcohol use disorders: a pilot study. Psychol Serv. 2012;9(2):174. doi:10.1037/ a0027606

23. Babor TF, Brown J, del Boca FK. Validity of self-reports in applied research on addictive behaviors: fact or fiction? Behavioral Assessment. 1990.

24. Simpson CA, Huang J, Roth DL, Chandler SD, Tucker JA. Predictors of utilization of an IVR self-monitoring program by problem drinkers with recent natural resolutions. Drug Alcohol Depend. 2012;126(1):111-117. doi:10.1016/j. drugalcdep.2012.04.022

25. Tucker JA, Roth DL, Huang J, Scott Crawford M, Simpson CA. Effects of interactive voice response self-monitoring on natural resolution of drinking problems: Utilization and behavioral economic factors. Journal of studies on alcohol and drugs. 2012;73(4):686-698. doi:10.15288/jsad.2012.73.686

26. Shiffman S, Stone AA, Hufford MR. Ecological momentary assessment. Annu Rev Clin Psychol. 2008;4:1-32. doi:10.1146/annurev.clinpsy.3.022806.091415

27. Finn PR, Rickert ME, Miller MA, Lucas J, Bogg T, Bobova L, et al. Reduced cognitive ability in alcohol dependence: examining the role of covarying externalizing psychopathology. J Abnorm Psychol. 2009;118(1):100. doi:10.1037/a0014656

28. Anstey KJ, von Sanden C, Salim A, O’Kearney R. Smoking as a risk factor for dementia and cognitive decline: a meta-analysis of prospective studies. Am J Epidemiol. 2007;166(4):367-378. doi:10.1093/aje/kwm116

29. Anthony JC, Echeagaray-Wagner F. Epidemiologic analysis of alcohol and tobacco use-Patterns of cooccurring consumption and dependence in the United States. 2013.

30. National Institute on Alcohol Abuse and Alcoholism. The National Institute on Alcohol Abuse and Alcoholism Five Year Strategic Plan: FY09-14. Alcohol Across the Lifespan. 2008.

31. Fiore M. Treating tobacco use and dependence: 2008 update. Clinical practice guideline. Diane Publishing; 2008.

32. Biener L, Abrams DB. The Contemplation Ladder: validation of a measure of readiness to consider smoking cessation. Health Psychol. 1991;10(5):360. doi:10.1037//0278-6133.10.5.360

33. Biener L, Abrams D. The contemplation ladder: A measure of intention to stop smoking. Health Psychol. 1991;10(5):360-365.

34. Amodei N, Lamb R. Convergent and concurrent validity of the Contemplation Ladder and URICA scales. Drug Alcohol Depend. 2004;73(3):301-306. doi:10.1016/j.drugalcdep.2003.11.005

35. Miller WR, Tonigan JS. Assessing drinkers' motivation for change: the Stages of Change Readiness and Treatment Eagerness Scale (SOCRATES). Psychol Addict Behav. 1996;10(2):81. doi:10.1037//0893-164x.10.2.81

36. Velicer WF, DiClemente CC, Rossi JS, Prochaska JO. Relapse situations and self-efficacy: An integrative model. Addict Behav. 1990;15(3):271-283. doi:10.1016/0306-4603(90)90070-e

37. Heatherton TF, Kozlowski LT, Frecker RC, Fagerstrom KO. The Fagerström test for nicotine dependence: a revision of the Fagerstrom Tolerance Questionnaire. Br J Addict. 1991;86(9):1119-1127.

38. First MB, Spitzer RL, Gibbon M, Williams JB. Structured Clinical Interview for DSM-IV Axis I Disorders, Patient Edition, January 1995 FINAL. SCID-I/P Version 2.0. New York, NY: Biometrics Research Department, New York State Psychiatric Institute;1995.

39. Sobell LC, Sobell MB. Timeline Followback user's guide: A calendar method for assessing alcohol and drug use. Toronto: Addiction Research Foundation; 1996.

40. World Health Organization. The Alcohol Use Disorders Identification Test. Guidelines for use in primary care. Geneva: World Health Organization; 2001.

41. Brown RA, Burgess ES, Sales SD, Whiteley JA, Evans DM, Miller IW. Reliability and validity of a smoking timeline follow-back interview. Psychol Addict Behav. 1998;12(2):101. doi:10.1037//0893-164x.12.2.101

42. Shiffman S, Hufford M, Hickcox M, Paty JA, Gnys M, 
Kassel JD. Remember that? A comparison of real-time versus retrospective recall of smoking lapses. J Consult Clin Psychol. 1997;65(2):292.

doi:10.1037/0022-006x.65.2.292.a

43. Kranzler HR, Abu-Hasaballah K, Tennen H, Feinn $\mathrm{R}$, Young $\mathrm{K}$. Using daily interactive voice response technology to measure drinking and related behaviors in a pharmacotherapy study. Alcoholism: Clinical and Experimental Research. 2004;28(7):1060-1064. doi:10.1097/01.alc.0000130806.12066.9c

44. Farkas AJ. When does cigarette fading increase the likelihood of future cessation? Ann Behav Med. 1999;21(1):71-76. doi:10.1007/bf02895036

45. Shaw RG, Mitchell-Olds T. ANOVA for unbalanced data: an overview. Ecology. 1993;74(6):1638-1645. doi:10.2307/1939922

46. Tucker JA. Natural resolution of alcohol-related problems. Recent developments in alcoholism. Springer; 2002:77-90.

47. Cohen J. The effect size index: f. Statistical power analysis for the behavioral sciences. 1988:284-288.

48. Tucker JA, Foushee HR, Black BC, Roth DL. Agreement between prospective interactive voice response selfmonitoring and structured retrospective reports of drinking and contextual variables during natural resolution attempts. Journal of studies on alcohol and drugs. 2007;68(4):538-542. doi:10.15288/jsad.2007.68.538

49. Centers for Disease Control and Prevention. Behavioral Risk Factor Surveillance System (BFRSS). http://www. cdc.gov/tobacco/data_statistics/index.htm. Published, 2014. Accessed September 13, 2016.

50. Perrine M, Mundt JC, Searles JS, Lester LS. Validation of daily self-reported alcohol consumption using interactive voice response (IVR) technology. J Stud Alcohol. 1995;56(5):487-490. doi:10.15288/jsa.1995.56.487

51. Mundt JC, KelleherPF, Perrine M, Searles JS. Psychological performance assessment via interactive voice response systems. Behav Res Methods. 1997;29(4):506-518. doi:10.3758/bf03210602

ACKNOWLEDGEMENTS

We would like to acknowledge the assistance of Drs Stephen Armeli and Thomas Brandon, who consulted on this grant and helped with protocol development and deployment.

CONFLICTS OF INTEREST Authors have completed and submitted the ICMJE Form for Disclosure of Potential Conflicts of Interest and none was reported.

FUNDING

This study was funded by grant R03CA 175870-01A1.

PROVENANCE AND PEER REVIEW

Not commissioned; externally peer reviewed 